\title{
Sonography in the instability of the long head of the biceps tendon confronted with histopathologic and arthroscopic findings
}

\author{
J. Zabrzyński ${ }^{1}$, Ł. Paczesny² ${ }^{2}$ A. Zabrzyńska ${ }^{3}$, D. Grzanka ${ }^{4}$, Ł. Łapaj ${ }^{5}$ \\ ${ }^{1}$ Department of Orthopaedic Surgery, Multidisciplinary Hospital, Inowroclaw, Poland \\ 2Department of Orthopaedic Surgery, Orvit Clinic, Torun, Poland \\ ${ }^{3}$ Department of Radiology, Multidisciplinary Hospital, Inowroclaw, Poland \\ ${ }^{4}$ Department of Clinical Pathomorphology, Nicolaus Copernicus University, Bydgoszcz, Poland \\ ${ }^{5}$ Department of General Orthopaedics, Musculoskeletal Oncology and Trauma Surgery, \\ Poznan University of Medical Sciences, Poznan, Poland
}

[Received: 1 November 2017; Accepted: 16 January 2018]

Background: Disorders of the long head of the biceps (LHB) tendon are a common source of shoulder pain and disability. This tendon can be well visualised using ultrasonography; however, little is known if such examination allows clinicians to predict pathological changes of the tendon structure. In the study described below, we compare preoperative sonographic findings with the data from shoulder arthroscopy and microscopic examination of the excised tendon fragments in 19 consecutive patients with LHB tendinopathy and clinical suspicion of its instability. Materials and methods: Preoperative ultrasonographic (US) inspection assessed several features of the tendon, whereas its stability was verified arthroscopically. In all cases, tenodesis or tenotomy procedures were performed and excised tendon fragments were harvested for microscopic examination based on the semiquantitative Bonar score.

Results: The most common US findings were hypoechoic areas, tendon thickening, an increased power Doppler signal and mechanical instability. Just as shoulder arthroscopy confirmed all mechanical instability cases detected in US, microscopic assessment revealed advanced degeneration in all samples.

Conclusions: Our study indicates that US is a useful tool in identifying cases of advanced instability and LHB tendinopathy, whereas biceps tendon instability is a biomechanically complex, gradually progressing phenomenon, frequently associated with additional shoulder lesions. (Folia Morphol 2018; 77, 3: 583-590)

Key words: tendinopathy; shoulder joint; biceps tendon

\section{INTRODUCTION}

Disorders of the long head of the biceps tendon (LHBT) are increasingly recognised as a common source of shoulder pain and disability. Although many classifications of LHBT pathologies exist, three main groups are commonly distinguished: inflammation, instability and trauma [10]. Data from numerous studies demon- strated that isolated LHBT injuries are typically found in young athletes competing in javelin throw and are rather uncommon in general population. Nevertheless, there is a large group of patients in whom LHBT lesions are associated with other shoulder pathologies, typically rotator cuff tears; some studies demonstrated strong correlations between these disorders $[1,10]$. 
It was found that a wide spectrum of mechanical pathologies can affect the LHBT, including instability, subluxation, luxation and tears. All of them may lead to tendinopathy - a degenerative process of tendinous tissue, often accompanied by pain in the anterior shoulder area. LHB tendinopathy is usually considered as an "overuse syndrome" caused by micro-injuries during everyday shoulder use. Some authors also suggested that the evolutionary movement of the scapula and the resulting compressing forces of the glenohumeral joint structures as well as glenohumeral arthritis contribute to LHBT damage $[1,7,10,21]$.

Understanding LHBT anatomy is important in the diagnostics of its lesions. The biceps tendon originates from the superior portion of the glenoid labrum and the supraglenoid tubercle of the scapula and can be divided into two portions: extracapsular and intracapsular. The tendon is stabilised by the transverse humeral ligament (THL), a tissue covering the bicipital groove [18]. Historically the THL was considered to be a primary biceps tendon stabiliser, formed by a broad band of fibrous tissue between lesser and greater tuberosity of the humerus; however, according to more recent studies, it is not a separate entity, but the structure formed mainly by the tendinous tissue originating from the subscapularis tendon (SSC), supraspinatus tendon (SST) and posterior lamina of pectoralis major muscle $[6,10,11,18]$.

The LHBT plays a role in humeral head depression, restrain of external rotation of the abducted arm and is a weak abductor of the shoulder; still, the knowledge of its biomechanics is limited $[1,10]$. Advancements in shoulder arthroscopy emphasized the importance of LHB tendinopathy in the development of shoulder pain and contributed to a wide use of tenotomy and tenodesis in its management [4]. Microscopic studies of pathological LHBT demonstrated the presence of a degenerative process without pronounced inflammation. These structural alterations affect the tendon appearance in ultrasonography (US), while the power Doppler (PD) function allows for the visualisation of neovascularisation $[8,15]$. The most common sonographic findings in biceps tendinopathy are: alterations of the fibrillar pattern with diffuse or focal hypoechogenicity, the presence of fluid in the bicipital groove, the thickening of the tendon, the presence of calcifications and the pathological vascularisation or dislocation of the tendon $[6,16]$. Despite the advances in imaging techniques, the role of ultrasound in the diagnosis of LHBT pathology is still unclear and constitutes a subject of debate $[2,19]$.

Our study compares the findings of preoperative sonographic assessment, shoulder arthroscopy and microscopic examination of the LHBT in patients in whom tendon instability accompanied by concomitant shoulder lesions was diagnosed. We wanted to evaluate the role of US in the diagnostics of LHBT instability and determine if sonographic findings make it possible to confirm the presence of structural alterations caused by tendinopathy.

\section{MATERIALS AND METHODS}

\section{Study group}

The study included 19 consecutive patients diagnosed with LHB tendinopathy and its instability based on physical examination and non-contrast shoulder magnetic resonance imaging (MRI). The mean age of the subjects was 54 (range 39-65); the cohort included 11 males and 8 females. In most patients LHB tendinopathy was diagnosed in association with various concurrent lesions of the affected shoulder: rotator cuff tears (RCTs) were found in 17 patients, while subacromial impingement (SI) was identified in 7 cases. Patients with rheumatic diseases, previous surgical treatment or corticosteroid injections in the past 12 months were excluded. The study was approved by the local bioethics committee (approval no. KB 598/2016), all patients were volunteers and gave informed consent.

\section{Sonographic examination}

All patients underwent ultrasound examination prior to arthroscopic treatment. Preoperative US examination was performed in the Orthopaedic Department by two orthopaedic surgeons experienced in musculoskeletal sonography, 1 or 2 days before the arthroscopic procedure; the examiners were blinded to any previous imaging data. US scans were performed using a linear $13 \mathrm{MHz}$ transducer using Esaote My Lab Gamma and Samsung Accuvix A30 systems. The protocol included only LHBT examination, in the sitting position, with the shoulder in $10^{\circ}$ of internal rotation, supinated elbow flexed to $90^{\circ}$, and the forearm resting on the lap. Short axis and long axis scans of the LHBT were taken: the probe was placed at the level of the bicipital groove, between lesser and greater tuberosity, and slightly moved upwards to gain axial LHBT scans; next, the transducer was orientated perpendicularly, between the tuberosities, to obtain longitudinal scans [6]. A part of the intracapsular portion (above the entrance to the intertubercular groove and inside the intertubercular groove) was visualised on axial and longitudinal sonograms. To obtain good quality images and eliminate the anisotropy effect, the probe was adjusted to be parallel to the tendon for both the transverse and longitudinal 
views [9]. The routine examination included PD imaging (increased colour flow signals in the biceps tendon area were recognised as an essential increase in neovascularisation). Afterwards, dynamic assessment of tendon stability and investigation of the shape of the bicipital groove were performed (the subchondral discontinuity or erosion on the humeral head were considered as essential indicators of tendon instability) [22].

During each examination the presence of 7 welldefined sonographic parameters was documented: the transverse diameter of the LHBT in the bicipital groove (normal diameters were $<4.6 \mathrm{~mm}$ for females and $<5.5 \mathrm{~mm}$ for males, as described by Huang et al. [9]), the presence of local alterations in tendon echogenicity in both longitudinal and transverse views, the presence of fluid around the tendon (more than $1 \mathrm{~mm}$ ), the presence of vascular signal within the tendon in the PD mode, the location of the tendon in the bicipital groove during the dynamic test, the presence of the "chondral print" sign [16, 22].

\section{Shoulder arthroscopy and microscopic examination}

All patients underwent shoulder arthroscopy performed in the beach chair position, in general anaesthesia; the operating surgeon was not blinded to the ultrasound results. The standard posterior portal and additional working portals were used; the shoulder was inspected for concomitant lesions [1]. The intracapsular part of the LHBT was tested for instability using an arthroscopic probe and examined for the presence of tears, excessive vascular injection, swelling, fraying, from its origin in the superior labrum to the bicipital groove. The area of the bicipital groove was inspected for the presence of biceps pulley lesions and possible chondral alterations. Later, the tendon was grasped using a dedicated probe and the extracapsular was pulled to the joint cavity for additional inspection.

In all cases tendon pathologies were confirmed arthroscopically and tenodesis or tenotomy procedures were performed and followed by the excision of the intracapsular part of the LHBT. The excised sample extended from the bicipital groove up to about $1 \mathrm{~cm}$ below the origin point at the supraglenoid tubercle (Fig. 1C). Next, this residual part was removed with the use of vaporisation technique. The resected tendon fragments (19 samples) were marked, fixed in sterile $10 \%$ buffered formalin and sent to the Pathology Department. After routine embedding in paraffin and mounting on slides, the samples were stained using the haematoxylin and eosin (H\&E) protocol and examined using light micros-

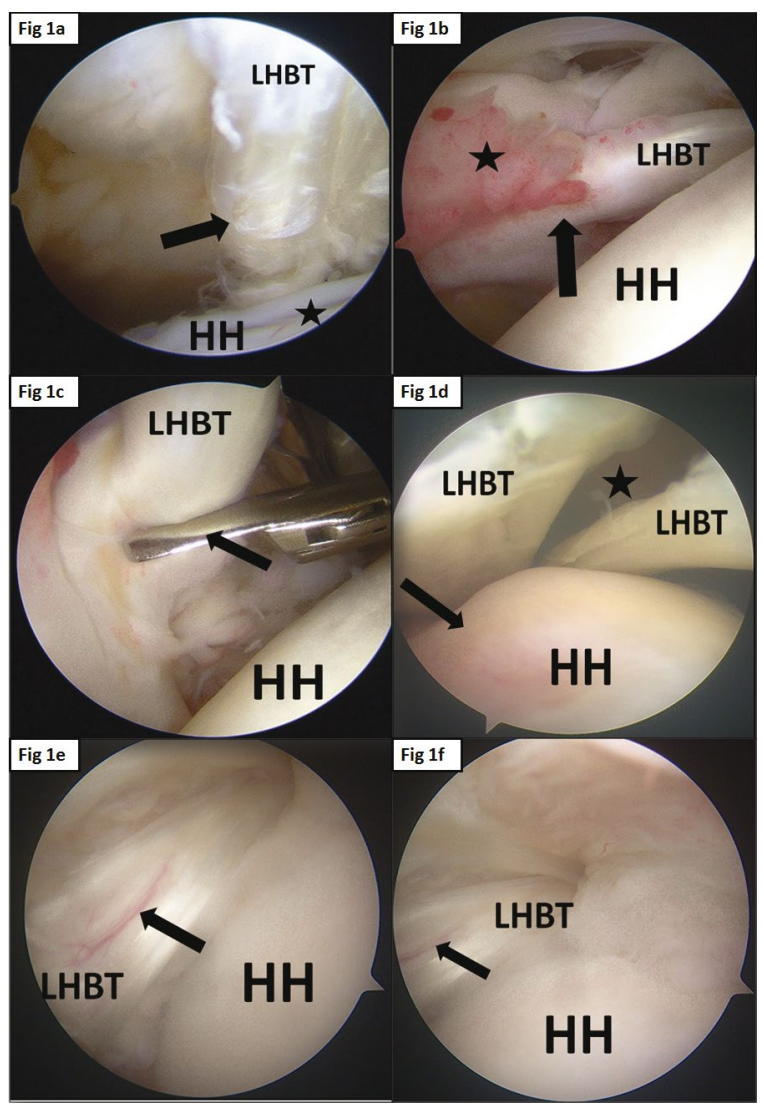

Figure 1. Arthroscopic pictures (posterior portal, $30^{\circ}$ arthroscope) illustrating; A. Typical partial tear (arrow) of the long head of the biceps tendon (LHBT) in the course of tendinopathy, thickened, flattened tendon structure and chondral abnormalities (asterisk) on the humeral head due to instability of the tendon in the intertubercular groove - "chondral print"; B. Anchor of the LHBT (arrow) to the superior labrum with synovitis (asterisk); C. The procedure of tenotomy in the area of the LHBT insertion using arthroscopic scissors (arrow); D. Pathological separation of the LHBT (significant tear) - asterisk, flattening of the structure and chondral abnormality on the humeral head; E, F. Vascular pattern - hyperaemia, on the surface of the LHBT - suggesting pathological process (arrow); $\mathrm{HH}$ - humeral head.

copy (Olympus BX46, Tokyo, Japan) by an experienced pathologist. Microscopic lesions were assessed using the Bonar scale, similar as in other studies regarding tendon pathologies $[13,17]$. This semiquantitative system evaluates four main variables: tenocyte morphology, changes in the ground substance and its accumulation, neovascularity and collagen bundles architecture. For each variable 0 to 3 points were attributed $(0-$ normal tissue, 3-extreme pathology), which yields a total of 0 (normal tendon) to 12 points (most severe abnormality possible). The microscopic analysis included the intracapsular part of the LHBT and the upper part from the bicipital groove, where the border between extra and intracapsular parts is located. 


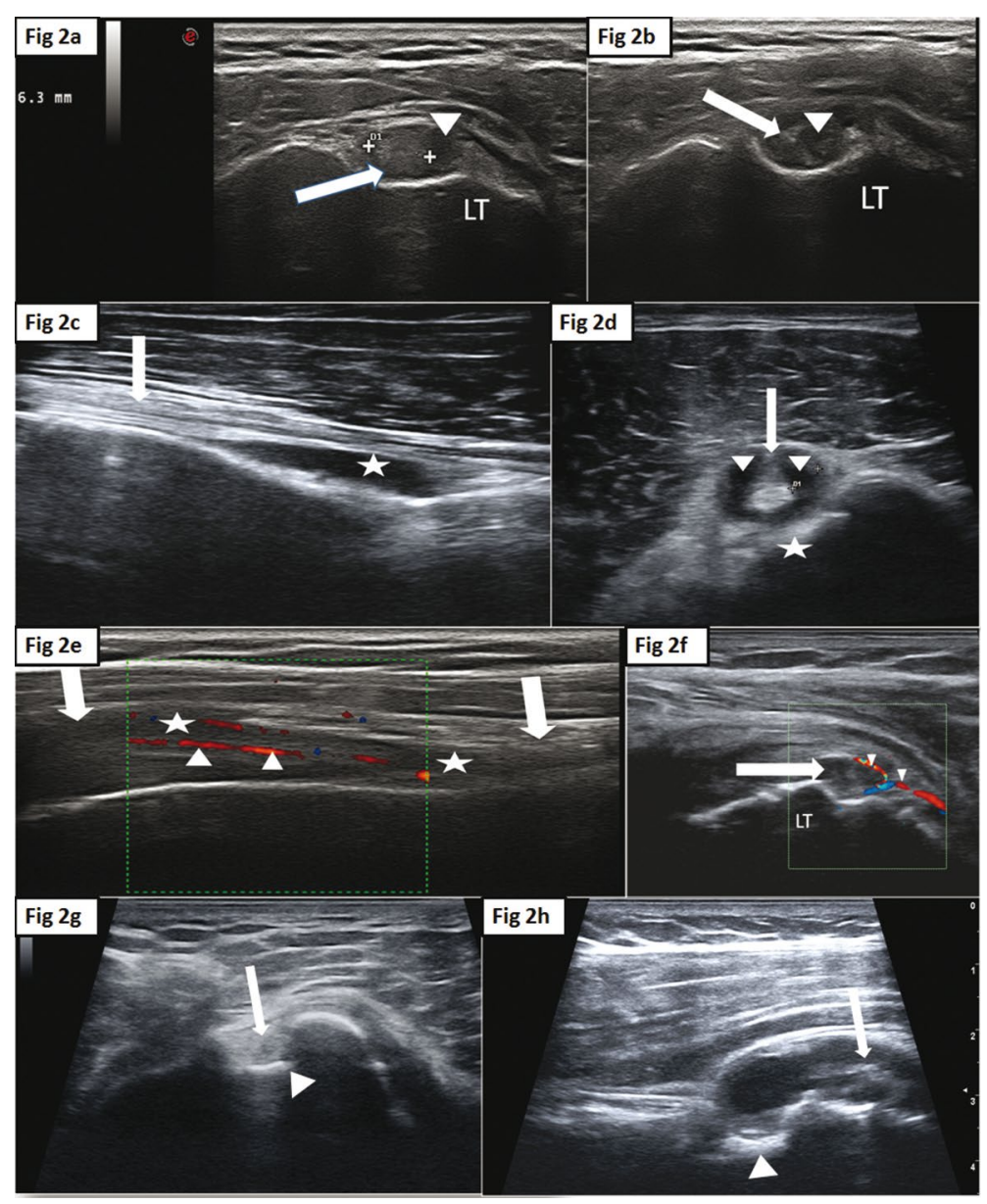

Figure 2. Sonographic pictures; A. Ultrasonographic (US) short-axis scan of the long head of the biceps tendon (LHBT) in the bicipital groove (arrow). The transverse diameter of the tendon is increased $-6.3 \mathrm{~mm}$, with hypoechogenic area suggesting the pathological fluid or synovial hypertrophy (arrowhead); B. US short-axis scan of the LHBT in the bicipital groove, characterised by smooth shape (arrow). The echogenicity of the LHBT is altered with centres of significantly decreased echogenicity (arrowhead); C. US long-axis scan of the LHBT (arrow). Asterisk indicates pathological accumulation of fluid between tendon and bicipital groove. The slightly, focally decreased echogenicity and dominantly characteristic fibrillar pattern of tendon structure is present; $\mathbf{D}$. US short-axis scan of the LHBT in the bicipital groove (arrow). The LHBT is correctly hyperechoic. Arrowheads indicate pathological accumulation of fluid and tenosynovitis whereas asterisk indicates deformation of the bicipital groove; E, F. US long-axis and short-axis scans of the LHBT (arrows). Increased Doppler signal implies the neovascularisation process in the tenosynovium of the LHBT and soft tissue in the area of the bicipital groove (arrowheads). The LHBT echogenicity is focally decreased suggesting the degeneration process (asterisk); G, H. US short-axis scan of the LHBT in the bicipital groove and luxated outside the bicipital groove (arrows). Arrowhead indicates deformation of the bicipital groove; LT — lesser tubercle.

Table 1. The summary of the sonographic examination of the long head of the biceps tendon (LHBT)

\begin{tabular}{lc}
\hline Sonographic features & Number of subjects \\
\hline Hypoechogenic areas & $18(95 \%)$ \\
Increased transverse LHBT diameter & $19(100 \%)^{*}$ \\
$(>5 \mathrm{~mm})$ measured in the groove & \\
Pathological fluid collection & $5(26 \%)$ \\
Increased blood flow signal in the power & $3(15 \%)$ \\
Doppler examination & $5(26 \%)^{* *}$ \\
Instability of tendon in the dynamic test & $2(10 \%)$ \\
\hline Presence of chondral print sign & \\
\hline
\end{tabular}

${ }^{*}$ Mean width $=6.15 \mathrm{~mm}$ (range 5-9 mm); ${ }^{*}$ In one case luxation beyond the groove

\section{RESULTS}

In most patients sonographic examination demonstrated the presence of several pathological features. The most common findings were an increased LHBT diameter and the presence of hypoechogenic areas (Table 1); they corresponded to the macroscopic lesions observed during the arthroscopic examination (Fig. 2A-H). The main arthroscopic pathological features of the LHBT were: hyperaemia, swelling, fraying, fibrillation and synovial overgrowth (Fig. 1A-F). Additional shoulder pathologies were visualised intraoperatively in most patients: RCTs in 17 cases 


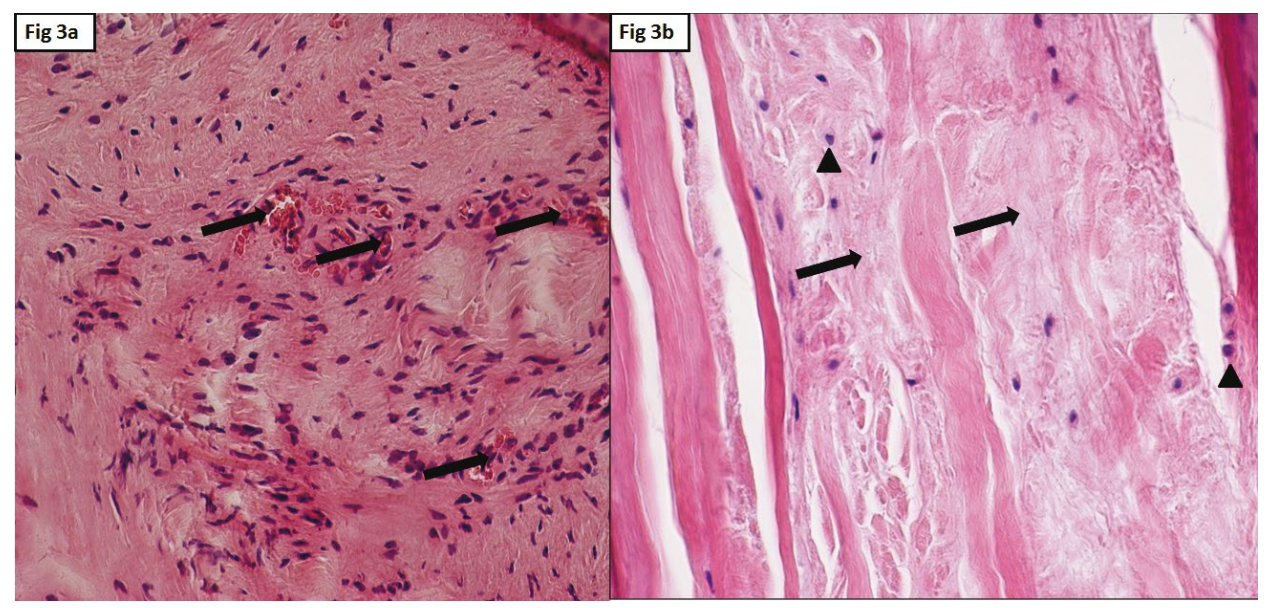

Figure 3. Microscopic pictures; A. Advanced vascular expansion with clusters of capillaries - arrows (stain: H\&E; magnification 200×); B. The accumulation of ground substance - arrows, the separation of collagen fibres by the ground substance, loss of characteristic "crimping pattern" and architecture of the collagen fibres; tenocytes with characteristic round shape - arrowheads (stain: H\&E; magnification 200×).

and $\mathrm{SI}$ and acromio-clavicular joint disorders in 7 cases. Partial-thickness tears and evident pulley lesions were less frequent and seen in 5 subjects. All LHBT instabilities detected in the prior ultrasound investigation were confirmed in arthroscopy, but no additional cases of instability were detected. The deformation of the bicipital groove and chondral lesions on the humeral head found during arthroscopy were consistent with the sonographic assessment. LHBT pathology was treated with tenotomy and tenodesis procedures in 5 and 14 subjects, respectively. The microscopic evaluation of the harvested tissues revealed advanced degeneration among all of the specimens with a mean Bonar score of 8.2 (range 4-11) (Fig. 3). Damaged tissue regions corresponded to the areas identified during the sonographic and arthroscopic examinations.

\section{DISCUSSION}

Long head of the biceps tendinopathy is a common condition in patients with shoulder pain and is often accompanied by RCTs, SI and other shoulder disorders [7, 14]. This fact makes the diagnostics of LHBT injuries difficult and often confounding, especially since there is no single clinical test which would provide an exact diagnosis and tears of the SST, the SSC can simulate symptoms of LHBT pathology $[7,20]$. This tendon is accessible by US; however, little is known if such examination enables predicting pathological changes of the tendon structure. The purpose of this study was to compare the sonographic appearance of the LHBT and its arthroscopic evaluation and microscopic changes. Several studies revealed limitations of contemporary imaging techniques in diagnosing LHBT condition. Although MRI is considered as a gold standard for the examination of soft tissue injuries, it was demonstrated to have low sensitivity in the detection of LHBT lesions [3, 10]. Similarly, the effectiveness of ultrasound examination in detecting LHBT pathologies is still unclear and the results provided by this type of examination depend on both the quality of the ultrasound system and the experience of the physician $[2,5,9,19]$. Skendzel et al. [19] emphasized the accuracy of US in the identification of normal, healthy LHBTs can be high (90\%). In their series, there were no cases of sonographically normal tendons presenting features of pathology during arthroscopic examination [19].

In this study, we demonstrated that sonographic examination can help predict instability and structural pathologies of the LHBT. Our study has several limitations, predominantly due to the fact that we included a relatively small group of patients with various types of concomitant shoulder lesions. This limits the impact of our results; however, other studies focused on microscopic and sonographic findings included a comparable number of patients with a variety of coexistent pathologies. Another limitation comes from the fact that we did not include samples of healthy tendons in our study for ethical reasons; therefore, we were unable to determine if the presence of sonographic features included in our protocol can be seen in well-functioning tendons without any pathological lesions. Consequently, the data from this paper are insufficient to determine the specificity of US examination in detecting LHB tendinopathy and 
LHBT instability. It should be noted that sonographic examination does not allow for the visualisation of the entire intracapsular part of the LHBT. Thus, we were unable conduct the imaging of some fragments of the tendon which were sent for microscopic examination; however, this limitation is inherent in all sonographic studies. Lastly, our results are most likely biased due to observer and device dependency; nevertheless, in order to minimise this, we used specifically defined criteria for sonographic features.

In all patients from this study there was good agreement between sonographic findings, clinical symptoms suggesting LHB tendinopathy and pathological findings which confirmed structural lesions. The sonographic appearance of the tendon is predominantly dependent on its microstructural alignment of collagen fibres, which affects the reflection of ultrasound waves corresponding to image intensity on the screen. Consequently, the distortion of the hierarchical tendinous structure observed in tendinopathy can result in decreased reflection of ultrasound waves within the parts of the tendon which are visible as hypoechoic areas. This was demonstrated by Huang et al. [9] who examined a series of patients suspected of LHB tendinopathy and quantified the grayscale data from two regions of interest in the transverse and coronal planes to determine tissue echogenicity. These authors were able to determine a threshold value, which corresponded with the clinical signs of tendinopathy; however, their study did not include arthroscopic and microscopic data. Our study demonstrated decreased echogenicity among almost all subjects and we believe that this was caused by structural lesions, similar as demonstrated in the studies including MRI examination $[3,10]$. It should be noted that concomitant pathologies found in most patients may have also affected the sonographic image to some degree.

Additionally, since most pathologic scoring systems include multiple morphologic features, there is no strong evidence which would indicate that US would allow determining the progression of tendinopathy. Still, our study clearly demonstrated that two sonographic features - hypoechoic areas and increased diameter of the tendon allow for identifying cases with advanced tendinopathy. This is especially important in case of patients, where multiple lesions make the diagnostics difficult, and may help in making clinical decisions regarding arthroscopic treatment.
The PD mode allowed us to detect pathological blood flow in the LHBT structure, which undeniably is a sign of a tendinopathy process; this finding is commonly seen and has been reported in previous studies $[12,15]$. Still, it should be noted that it is generally accepted that there is no link between mechanical instability and the neovascularisation process $[12,15]$.

An increased diameter of the tendon was a common finding in our cohort. Huang et al. [9] defined the thickness of tendon biceps in its tendinopathy in US transverse scans: $\geq 4.6 \mathrm{~mm}$ for women and $\geq 5.5 \mathrm{~mm}$ for men. It should be noticed that LHBT thickness also depends on age and sports activity $[9,16]$. Increased thickness is classified as non-tear abnormality and may be associated with oedema and the swelling of the tendon, which are symptoms of tendinopathy [18]. In our group, the transverse diameter of LHBT was in the range of 5 to $9 \mathrm{~mm}$, which can be considered as an indirect symptom of tendon pathology linked with its clinical instability.

Sonographic confirmation of effusion around the LHBT has historically been considered as a sign of an exacerbated inflammatory process within the tendon. It should be noted that although the LHBT has its intracapsular part, it lies extrasynovially and the bicipital groove communicates with the glenohumeral joint [16]. Consequently, several studies demonstrated that although fluid inside the bicipital groove is abnormal, this sign is non-specific for LHBT pathology. One study indicated that in $90 \%$ of cases it is associated with different pathologies of the glenohumeral joint and many authors accented the deceptive role of this sonographic feature in the diagnostics of tenosynovitis $[6,16]$. In our group, an abnormal amount of fluid was present in 5 cases, and it is possible that it was related to concomitant shoulder lesions: 3 patients had SST ruptures, while 2 had simultaneous SST and SSC ruptures.

Although sonographic examination enables conducting dynamic testing of tendon stability, features of sublocation or dislocation were rare in our group. This seems surprising since all tendons demonstrated the presence of tendinopathy and it is often suggested that this process is associated with overuse or instability [1]. It should be underlined that US allows for a very precise dynamic stability assessment during both external and internal rotation of the arm and the tendon transposition or dislocation can be well visualised using standard probes. Additionally, instability can be confirmed during a routine examination by the 
presence of a recently described "chondral print" sign [22]. Gross LHBT instability causes a deformation of the bicipital groove, an irregularity which exposes the tendon to subsequent injury develops and causes the splitting of condensed tendon structure. The presence of these features has always been associated with mechanical instability confirmed in arthroscopy, similar as reported in other studies where the sensitivity of the "chondral print" signs was as high as $90 \%[1,2]$. It has been suggested that instability could be split into an initial phase: instability associated with pulley lesions resulting in microscopic tendon migration and a subsequent advanced phase characterised by macroscopic instability resulting in subluxation or luxation and the development of microstructural lesions [22]. This could explain why US features of instability were rare in our group although features of tendinopathy were seen in all subjects and the RCTs were present in almost $90 \%$ of cases. The THL formed by fibres of the SST and SSC, is often damaged due to RCTs with subsequent biceps tendon stability disorders [18]. Therefore, we conclude that micro-instability, which leads to gradual development of tendinopathy, may not be detected in an US examination. However, it may gradually progress (potentially becoming exacerbated by the RCTs) to a point when subluxation or dislocation may be detected during a dynamic US examination. Still, on the basis of the data from our study we cannot determine how various degrees of instability affect structural lesions of tendinous tissue.

It is generally accepted that the role of different imaging modalities in the diagnostics of LHBT pathologies is limited. Although the MRI can provide both excellent visualisation of the tendon and indicate alterations in the tissue structure, multiple authors demonstrated low sensitivity for detecting partial thickness tears and instability $[3,10]$. Other authors indicated that US of the LHBT seems to be a good method for confirming instability, subluxation, luxation and full-thickness tears, despite poorer visualisation of the tendinous structure $[2,19]$. Similarly, our study demonstrated that US can be a valuable tool in confirming gross LHBT instability and identifying the presence of tendinopathy.

\section{CONCLUSIONS}

Sonography of the biceps tendon is useful in detecting lesions related to different stages of instability and can demonstrate the presence of intra-tendinous pathologies which could otherwise be missed during an arthroscopic examination. Even though ultrasound examination is limited due to its subjective nature, we demonstrated that certain findings, such as an increased tendon diameter or the presence of hypoechogenic regions, allow for identifying cases of LHB tendinopathy, which may be associated with tendon micro-instability. However, since LHBT instability is a biomechanically complex, gradually progressing phenomenon, in such cases, the clinical decision cannot be made on sonographic findings and should be also based on clinical examination supported by other imaging techniques.

\section{REFERENCES}

1. Ahrens PM, Boileau P. The long head of biceps and associated tendinopathy. J Bone Joint Surg Br. 2007; 89(8): 1001-1009, doi: 10.1302/0301-620X.89B8.19278, indexed in Pubmed: 17785735.

2. Armstrong A, Teefey SA, Wu T, et al. The efficacy of ultrasound in the diagnosis of long head of the biceps tendon pathology. J Shoulder Elbow Surg. 2006; 15(1): 7-11, doi: 10.1016/j.jse.2005.04.008, indexed in Pubmed: 16414462.

3. Carr RM, Shishani $Y$, Gobezie R. How accurate are we in detecting biceps tendinopathy? Clin Sports Med. 2016; 35(1): 47-55, doi: 10.1016/j.csm.2015.08.002, indexed in Pubmed: 26614468.

4. Ditsios K, Agathangelidis F, Boutsiadis A, et al. Long head of the biceps pathology combined with rotator cuff tears. Adv Orthop. 2012; 2012: 405472, doi: 10.1155/2012/405472, indexed in Pubmed: 23209915.

5. Ferrari FS, Governi S, Burresi F, et al. Supraspinatus tendon tears: comparison of US and MR arthrography with surgical correlation. Eur Radiol. 2002; 12(5): 1211-1217, doi: 10.1007/s00330-001-1183-3, indexed in Pubmed: 11976869.

6. Fodor D. Ultrasonography of the normal and pathologic long head of biceps tendon. Medical Ultrasonography. 2009; 11(2): 45-51.

7. Gill HS, El Rassi G, Bahk MS, et al. Physical examination for partial tears of the biceps tendon. Am J Sports Med. 2007; 35(8): 1334-1340, doi: 10.1177/0363546507300058, indexed in Pubmed: 17369556.

8. Gisslén $\mathrm{K}$, Alfredson $\mathrm{H}$. Neovascularisation and pain in jumper's knee: a prospective clinical and sonographic study in elite junior volleyball players. Br J Sports Med. 2005; 39(7): 423-8; discussion 423, doi: 10.1136/ bjsm.2004.013342, indexed in Pubmed: 15976162.

9. Huang SW, Wang WT. Quantitative diagnostic method for biceps long head tendinitis by using ultrasound. ScientificWorldJournal. 2013; 2013: 948323 , doi: 10.1155/2013/948323, indexed in Pubmed: 24385888.

10. Khazzam M, George MS, Churchill RS, et al. Disorders of the long head of biceps tendon. J Shoulder Elbow Surg. 2012; 21(1): 136-145, doi: 10.1016/j.jse.2011.07.016, indexed in Pubmed: 22005126.

11. Lee $M H$, Sheehan SE, Orwin JF, et al. Comprehensive Shoulder US Examination: A Standardized Approach with 
Multimodality Correlation for Common Shoulder Disease. Radiographics. 2016; 36(6): 1606-1627, doi: 10.1148/ rg.2016160030, indexed in Pubmed: 27726738.

12. Lewis JS, Raza SA, Pilcher J, et al. The prevalence of neovascularity in patients clinically diagnosed with rotator cuff tendinopathy. BMC Musculoskelet Disord. 2009; 10: 163, doi: 10.1186/1471-2474-10-163, indexed in Pubmed: 20025761

13. Maffulli N, Longo UG, Franceschi F, et al. Movin and Bonar scores assess the same characteristics of tendon histology. Clin Orthop Relat Res. 2008; 466(7): 1605-1611, doi: 10.1007/s11999-008-0261-0, indexed in Pubmed: 18437501.

14. Murthi AM, Vosburgh CL, Neviaser TJ. The incidence of pathologic changes of the long head of the biceps tendon. J Shoulder Elbow Surg. 2000; 9(5): 382-385, doi: 10.1067/ mse. 2000.108386, indexed in Pubmed: 11075320.

15. Alfredson $H$, Ohberg L, Zeisig E, et al. Neovascularisation in Achilles tendons with painful tendinosis but not in normal tendons: an ultrasonographic investigation. Knee Surg Sports Traumatol Arthrosc. 2001; 9(4): 233-238, doi: 10.1007/s001670000189, indexed in Pubmed: 11522081

16. Ptasznik R, Hennessy O. Abnormalities of the biceps tendon of the shoulder: sonographic findings. AJR Am
J Roentgenol. 1995; 164(2): 409-414, doi: 10.2214/ ajr.164.2.7839979, indexed in Pubmed: 7839979.

17. Singaraju VM, Kang RW, Yanke AB, et al. Biceps tendinitis in chronic rotator cuff tears: a histologic perspective. J Shoulder Elbow Surg. 2008; 17(6): 898-904, doi: 10.1016/j. jse.2008.05.044, indexed in Pubmed: 18786837

18. Singh R, Singla M, Tubbs RS. Macro/micro observational studies of fibres maintaining the biceps brachii tendon in the bicipital groove: application to surgery, pathology and kinesiology. Folia Morphol. 2015; 74(4): 439-446, doi: 10.5603/ FM.2015.0105, indexed in Pubmed: 26620503.

19. Skendzel JG, Jacobson JA, Carpenter JE, et al. Long head of biceps brachii tendon evaluation: accuracy of preoperative ultrasound. AJR Am J Roentgenol. 2011; 197(4): 942-948, doi: 10.2214/AJR.10.5012, indexed in Pubmed: 21940583.

20. Zabrzyński J, Szumlański A. Tendinopathy of the long head of the biceps tendon - physical examination confronted with shoulder arthroscopy findings. Med Biol Scien. 2016; 30(2): 67, doi: $10.12775 / \mathrm{mbs} .2016 .008$.

21. Zabrzyński J, Zabrzyńska A, Grzanka D. Tendinopathy a disease of tendons. J Orthop Trauma Surg Rel Res. 2016; 3(40): 024-030.

22. Zappia M, Carfora M, Romano AM, et al. Sonography of chondral print on humeral head. Skeletal Radiol. 2016; 45(1): 35-40, doi: 10.1007/s00256-015-2238-x, indexed in Pubmed: 26298103. 